\title{
Lutzomyia longiflocosa, posible vector en un foco de leishmaniasis cutánea en la región subandina del departamento del Tolima, Colombia, y el conocimiento que tiene la población sobre este insecto
}

\author{
Raúl H. Pardo ${ }^{1}$, Olga Lucía Cabrera ${ }^{1}$, Jorge Becerra ${ }^{2}$, \\ Patricia Fuya ${ }^{1}$, Cristina Ferro ${ }^{1}$ \\ ${ }^{1}$ Laboratorio de Entomología, Instituto Nacional de Salud, Bogotá, D.C, Colombia \\ 2 Unidad de Entomología, Secretaría de Salud del Tolima.
}

Introducción. Durante los años 2003 y 2004 se presentó en el área rural de los municipios de Chaparral y San Antonio, sobre la región subandina del departamento del Tolima, lo que parece ser la epidemia más grande (2.810 casos) de leishmaniasis cutánea en Colombia, con una incidencia máxima de $6.202 \times 100.000$ en el año 2004.

Objetivo. El presente estudio se realizó con el objeto de aportar evidencias para incriminar al vector y establecer el conocimiento que los habitantes tienen de los flebótomos para aplicar esta información en el control vectorial.

Materiales y métodos. Se muestrearon 46 viviendas con trampas de luz tipo CDC para identificar las especies de flebótomos, su abundancia y porcentajes de infestación intradomiciliar. Durante el día se revisaron las viviendas para determinar el comportamiento endofílico. Se aplicó una encuesta para determinar el conocimiento que la población tenía sobre el vector, su papel en la transmisión y los sitios y épocas de abundancia.

Resultados. Tres especies antropofílicas con posible importancia epidemiológica fueron encontradas. L. longiflocosa fue la especie dominante en el $81,7 \%$ (192 / 235) de las capturas e infestó el mayor número de viviendas, $41,7 \%$. Le siguieron $L$. columbiana y $L$. nuneztovari con abundancias relativas de $3,4 \%$ y $2,1 \%$, respectivamente, e infestaciones de $13,0 \%$ y $6,5 \%$, respectivamente. No se encontró comportamiento endofílico. Los habitantes entrevistados conocían al vector y su papel en la transmisión, señalando a la vivienda y al verano como el sitio y la época de mayor abundancia.

Conclusiones. Por su notable dominancia y aparente endofagia, L. longiflocosa es el vector más probable de leishmaniasis en el intradomicilio. L. columbiana y L. nuneztovari podrían ser vectores secundarios en el extradomicilio. Las implicaciones de los resultados en el control vectorial son discutidas.

Palabras clave: Psychodidae, leishmaniasis cutánea, Colombia

Lutzomyia longiflocosa as suspected vector of cutaneous leishmaniasis in a focus of cutaneous leishmaniasis on the sub-andean region of Tolima department, Colombia, and the knowledge on sandflies by the inhabitants

Introduction. Between 2003 and 2004 the largest epidemic of cutaneous leishmaniasis in Colombia (2,810 cases, with the highest incidence of $6,202 \times 100,000$ in 2004) occurred in the sub-Andean rural area of the municipalities of Chaparral and San Antonio in the department of Tolima.

Objective. The present study was carried out to identify suspected vectors and to establish the knowledge that the inhabitants have about sand flies in order to use this information for vector control.

Materials and methods. 46 houses were sampled with CDC light traps set up indoors to establish the sand fly species composition, abundance and the percentage of infestation. Houses were examined during daylight to identify endophily. A questionnaire was applied in order to 
estimate the knowledge about sand flies, their role in transmission and the sites and seasons of highest abundance.

Results. Three anthropophilic sand fly species of possible epidemiological importance were found. L. longiflocosa was the dominant sand fly species accounting for $81.7 \%$ (192 / 235) of all catches and infested the highest number of houses $(41.7 \%)$. The other two species were $L$. columbiana and $L$. nuneztovari, with relative abundances of $3.4 \%$ and $2.1 \%$, respectively, and house infestations of $13.0 \%$ and $6.5 \%$, respectively. There was no evidence of endophilic behavior. Inhabitants recognized sand flies and their role in transmission. They identified the houses and the dry season as the site and time period of highest sand fly abundance.

Conclusions. Based on its high anthropophily, predominance and apparent endophagic behavior, L. longiflocosa is the most probable vector of leishmaniasis indoors. L. columbiana and $L$. nuneztovari could be involved as secondary vectors outdoors. The importance of these findings on sand fly control is discussed.

Key words: Psychodidae, cutaneous leishmaniasis, Colombia

De las tres formas clínicas de leishmaniasis conocidas, leishmaniasis cutánea, mucocutánea y visceral, la forma cutánea es la dominante en Colombia con el $95 \%$ de los casos. Desde que se inició la notificación obligatoria de la enfermedad en la década de los ochentas, la incidencia de la leishmaniasis ha presentado una tendencia al aumento en el país, con la excepción de los años 2000 y 2001 en los que hubo una notable reducción (registros epidemiológicos del Ministerio de la Protección Social).

Aunque este aumento ha sido atribuido a una mayor búsqueda y al mejor diagnóstico y notificación de la enfermedad (1), esto no parece ser suficiente para explicar el notable incremento de casos de leishmaniasis cutánea en los dos últimos años. De una incidencia con una mediana de 58,7 casos por 100.000 habitantes (total de casos: 57.807 ) en la década de los noventa, se pasó a una mediana de 89,5 casos por 100.000 en los años 2003 y 2004 (total de casos: 24.168) (cálculos con base en los registros epidemiológicos del Ministerio de la Protección Social).

En el departamento del Tolima, hasta el 2002, el reporte de casos de leishmaniasis cutánea había sido tradicionalmente bajo, con tendencia al

\footnotetext{
Correspondencia:

Raúl H. Pardo, Laboratorio de Entomología, Instituto Nacional de Salud, Bogotá, D.C., Colombia.

Teléfono: 454 4191; fax: 2200923

raulpardopuentes@yahoo.co.uk
}

Recibido: 1/08/05; aceptado: 24/02/06 aumento. En la década de los ochenta se reportaron 840 casos (mediana=67 casos/año), mientras que en la década de los noventa se registraron 1.833 casos (mediana=149 casos/año). Sin embargo, en 2003 y 2004 el registro de casos se incrementó drásticamente llegando a 3.094 casos (mediana $=1.547$ casos/año) (cálculos con base en los registros epidemiológicos del Ministerio de la Protección Social y del Sistema de Vigilancia en Salud Pública, SIVIGILA, del Instituto Nacional de Salud, INS), la mayoría originados en el área montañosa de los municipios contiguos a Chaparral y San Antonio, ubicados sobre la Cordillera Central.

En el 2004, Chaparral presentó la mayor incidencia de leishmaniasis cutánea en el país, 8.444 por 100.000, con 1.616 casos, mientras que San Antonio ocupó el tercer lugar con una incidencia de 2.389 por 100.000 (269 casos). El registro de Chaparral representa la mayor epidemia de leishmaniasis cutánea reportada en Colombia. Llama la atención que, históricamente, estos dos municipios no habían sido reconocidos como focos epidémicos de la enfermedad. Más aun, en Chaparral sólo se habían reportado esporádicamente casos de leishmaniasis cutánea (2).

Una descripción general de la epidemia de Chaparral y San Antonio la hicieron Morales et al. (2) quienes encontraron que: 1) la enfermedad se presentaba principalmente en el área rural, 96,8\% de los casos; 2) afectaba más a los hombres, $59,1 \%$ y $57,3 \%$ de los casos en Chaparral y San Antonio, respectivamente, y 3 ) los grupos de edad más comprometidos fueron los de edades más 
tempranas. Además, el grupo de edad de personas más jóvenes, de cero a cuatro años, presentó un número elevado de casos, aproximadamente, 180 casos en Chaparral y 50 casos en San Antonio. El compromiso de personas en edades tempranas permite plantear que, por lo menos, una parte importante de la transmisión podría estar presentándose en el intradomicilio o en el peridomicilio.

La información relacionada con parásitos, vectores y reservorios es muy limitada. Como agente etiológico se ha identificado hasta el momento a Leishmania panamensis, aislada de algunos pacientes (Ayala MS, Morales D, Nicholls RS. Aspectos parasitológicos de la epidemia de leishmaniasis en el Tolima, 2003-2004. Memorias, IX Encuentro Científico del Instituto Nacional de Salud, Ciencia, desarrollo y Salud: ayer, hoy y mañana. Bogotá, 2 y 3 de diciembre del 2004). Aunque en el foco de Chaparral y San Antonio no se ha identificado al vector, en dos áreas similares a este foco se ha involucrado a Lutzomyia longiflocosa como posible vector, una al sur del departamento, en un foco del municipio de Planadas (3) y la otra en el vecino departamento del Huila en el foco de leishmaniasis cutánea ubicado al nororiente del departamento sobre la Cordillera Oriental (Pardo R, Ferro C, Lozano G, Lozano C, Cabrera OL, Davies C. Flebótomos (Diptera: Psychodidae) vectores de leishmaniasis cutánea y sus determinantes ecológicos en la zona cafetera del departamento del Huila. Memorias, XXVI Congreso Sociedad Colombiana de Entomología, Bogotá, 28-30 de julio de 1999). En ambos focos se presentaron evidencias de comportamiento endofágico de esta especie, definido como el hábito de picadura dentro de construcciones humanas, lo cual indica que la transmisión puede estar ocurriendo en el intradomicilio.

Teniendo en cuenta que la presencia de $L$. longiflocosa ha sido reportada en cinco municipios del Tolima, Herveo (4), Casabianca (datos sin publicar del Laboratorio de Entomología, INS), Rovira (5), Ortega (6) y Planadas (3) a lo largo de la Cordillera Central, y la evidencia epidemiológica que sugiere una transmisión intradomiciliaria, se plantea que, en el foco de Chaparral y San Antonio,
L. longiflocosa también podría estar involucrada como vector.

Dada la gravedad de la epidemia en Chaparral y San Antonio se hizo prioritario tomar medidas de control vectorial de emergencia; por esta razón, a finales de 2003 y principios de 2004, la Secretaría de Salud del Tolima realizó la fumigación con lambdacihalotrina (ICON) en las viviendas de las veredas con mayor incidencia de la enfermedad. Sin embargo, la falta del conocimiento de la especie vectora y de aspectos fundamentales de su biología, como los hábitos de endofagia y endofilia, hicieron necesario realizar un estudio entomológico básico a corto plazo que respaldara el control vectorial aplicado o permitiera plantear la introducción de medidas alternativas como el uso de toldillos impregnados con insecticida.

El estudio permitió evaluar el posible papel vectorial de las especies de flebótomos encontradas, con base en la determinación de su abundancia intradomiciliaria, los porcentajes de infestación de las viviendas y el comportamiento endofílico (definido como el hábito de reposar en construcciones cubiertas hechas por humanos durante, al menos, una parte del ciclo gonadotrófico) (7). Como complemento, se indagó sobre el conocimiento que tenían los habitantes del área de estudio del vector y de su papel en la transmisión y sobre la aceptación que podría tener la introducción de toldillos impregnados con insecticida.

\section{Materiales y métodos \\ Descripción del área}

El estudio se realizó entre el 7 y el 15 de julio de 2004 en los municipios contiguos a Chaparral y San Antonio, ubicados sobre el flanco oriental de la Cordillera Central, al suroccidente del departamento del Tolima, el cual hace parte de la cuenca alta del río Magdalena.

En cada municipio se seleccionó para el trabajo una de las veredas de alta incidencia de leishmaniasis cutánea (según los registros epidemiológicos, 2003-2004, de la Secretaría de Salud Departamental del Tolima) (figura 1); la mayoría se ubican, aparentemente, dentro de la región subandina (1.000-2000 msnm). 


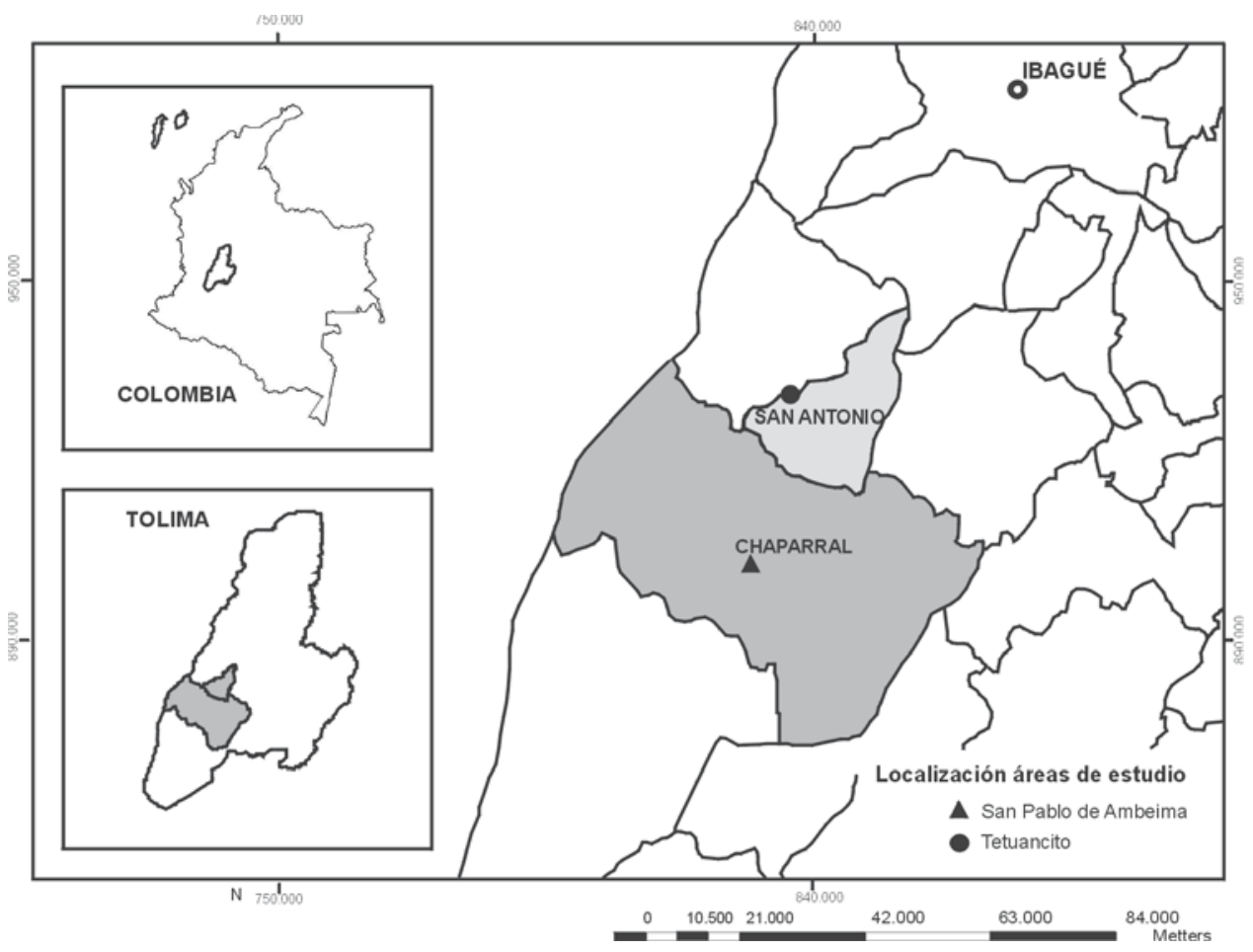

Figura 1. Localización de las veredas incluidas en el estudio del foco de leishmaniasis cutánea de los municipios de Chaparral y San Antonio en el departamento del Tolima.

En el municipio de Chaparral se seleccionó la vereda San Pablo de Ambeima que registró una incidencia de 310 casos por 1.000 habitantes. Esta vereda se ubica a los $3^{\circ} 44^{\prime} \mathrm{N}$ y $75^{\circ} 34^{\prime} \mathrm{O}$ con una variación altitudinal aproximada entre 1.300 y $1.700 \mathrm{msnm}$; la temperatura promedio calculada es de $21,5^{\circ} \mathrm{C}$, y la precipitación anual promedio es de $2.607 \mathrm{~mm}$ (estación El Limón, código: 2204502, datos 2003, IDEAM).

En el municipio de San Antonio se trabajó en la vereda Tetuancito, en donde la incidencia de leishmaniasis cutánea fue de 110 casos por 1.000 habitantes. Esta vereda se localiza a $3^{\circ} 58^{\prime} \mathrm{N}$ y $75^{\circ} 33^{\prime} \mathrm{O}$ con una variación altitudinal entre 1.600 y $2.000 \mathrm{msnm}$; la temperatura promedio es de $21^{\circ} \mathrm{C}$, y la precipitación anual de $1.985 \mathrm{~mm}$ (estación San Antonio, código: 2206504, datos 2003, IDEAM).

La distancia entre las dos veredas es de $55 \mathrm{~km}$, aproximadamente. Ambas veredas están sometidas a un régimen climático bimodal con dos épocas de verano, la primera de diciembre a febrero y la segunda de junio a agosto. La economía se basa, principalmente, en la agricultura (cultivo de café, fríjol y otros) y en la producción pecuaria.

\section{Muestreo de flebótomos}

La determinación de la abundancia intradomiciliaria y el porcentaje de infestación de cada especie de flebótomo se hizo mediante el uso de trampas de luz tipo CDC, colocadas entre las 18:00 y las 06:00 horas, en uno de los dormitorios de cada vivienda, por una sola vez. Se muestrearon 46 viviendas: 22 viviendas en San Pablo de Ambeima y 24 viviendas en Tetuancito, que corresponden a $48 \%$ y $43 \%$ de las viviendas en cada vereda, respectivamente.

Para la selección de las viviendas se tuvo en cuenta que en estas hubiera reporte de casos de leishmaniasis cutánea y que no hubieran sido fumigadas con insecticidas de acción residual, por lo menos, durante los cuatro meses previos 
al muestreo. El establecimiento de un tiempo mayor, deseable para evitar el efecto residual de la fumigación, no fue posible debido a que para el momento del estudio la mayoría de las veredas habian sido fumigadas con lambdacihalotrina como respuesta a la gravedad de la epidemia. Aunque el efecto residual de este insecticida sobre los flebótomos puede durar, por lo menos, hasta seis meses (8), se espera que su impacto sobre L. longiflocosa, especie no endofílica, que podría estar presente y ser el vector en el área de estudio, sería muy limitado o no tener ninguno como se demostró en un estudio en el departamento del Huila (Pardo R, Ferro C, Davies C. Efficacy and efectiveness of insecticide treated bednets in Colombia. 5th International Symposium on Phlebotominae sandflies (ISOPS 5), Tunisia, April 17-21, 2005). El muestreo de viviendas con reporte de casos de leishmaniasis cutánea se hizo porque en viviendas con estas características hay una alta probabilidad de captura de L. longiflocosa.

Como complemento al muestreo intradomiciliario se colocaron trampas CDC a 1,5 m de altura, entre las 18:00 y las 07:00 horas en algunos sitios extradomiciliarios, registrando el tipo de hábitat: a) bosque; b) cafetales tradicionales, en donde un estrato arbóreo proporciona un notable sombrío a las plantas de café; c) cafetal al sol, en donde hay muy poco sombrío o no existe; d) pastizal, y e) rastrojo.

Para confirmar la antropofilia de los posibles vectores se hicieron dos muestreos con cebo humano en el extradomicilio y uno en el intradomicilio entre las $18: 00$ y las $21: 00$ horas. La captura fue hecha por dos personas que se protegieron con prendas adecuadas. Los flebótomos se capturaron tan pronto como se posaban sobre los personas, sin darles tiempo a picar.

El comportamiento endofílico se determinó mediante la búsqueda de flebótomos durante las horas del día en todas las viviendas incluidas en el muestreo. Dos personas dotadas con aspiradores de boca y linterna muestrearon los posibles sitios de reposo (paredes internas y externas de las viviendas, gallineros, marraneras y otras construcciones). Los flebótomos capturados se identificaron siguiendo las claves de Young y Duncan (5).

\section{Conocimientos sobre el vectory opinión sobre el uso de toldillos}

La determinación del conocimiento que tienen los habitantes del área de estudio del vector se realizó mediante una encuesta escrita aplicada por una persona previamente entrenada, al jefe de familia, cónyuge $o$, de no estar ellos, a una persona mayor de 14 años, en las mismas viviendas del muestreo entomológico. Los temas incluidos en la encuesta fueron: 1) reconocimiento del insecto vector; 2 ) conocimiento de su papel en la transmisión de la leishmaniasis cutánea; 3 ) identificación de sitios de alta abundancia vectorial; 4) identificación de las épocas de alta abundancia vectorial, y 5) opinión sobre el posible uso de toldillos impregnados para el control vectorial (aplicado sólo en la vereda San Pablo de Ambeima).

Para determinar si las personas entrevistadas conocían al insecto vector, se diseñó una pregunta en la que se describían cinco características del vector: a) dos de morfología, tamaño menor comparado con un zancudo y alas "paradas", en forma de V, cuando el insecto está en reposo; b) una de desplazamiento, vuelo corto a saltos, y c) dos sobre la picadura y sus consecuencias inmediatas, picadura como "quemón" o "pringue" y "mancha" o "pinta colorada" como consecuencia de la misma. Se consideró que el reconocimiento, por lo menos, de dos de las anteriores características era suficiente para aceptar que el entrevistado conocía al vector.

Todos los entrevistados aceptaron participar voluntariamente tanto en la encuesta como en permitir el muestreo entomológico.

\section{Análisis de la información}

El análisis estadístico de los datos entomológicos se hizo sobre la abundancia de las hembras de $L$. longiflocosa en el intradomicilio, los porcentajes de infestación en las viviendas de cada una de las especies encontradas y sobre las frecuencias de las variables estudiadas en la encuesta de conocimientos del vector.

La abundancia intradomiciliaria de L. Iongiflocosa se presenta en los cuadros como promedios 
geométricos de Williams, del número de hembras /trampa CDC/noche $(\mathrm{h} / \mathrm{CDC} / \mathrm{n})$ con sus intervalos de confianza del $95 \%$. El análisis de abundancia se hizo usando modelos lineales generalizados (GLIM), asumiendo que los datos en crudo seguían la distribución binomial negativa, usando el programa estadístico Stata 7. La comparación de abundancia entre hábitats se hizo teniendo en cuenta las diferencias geográficas por vereda.

Los porcentajes de infestación de las viviendas se calcularon para cada especie con base en la fórmula:

Número de viviendas con presencia de flebótomos

Número total de viviendas $X 100$

El análisis de estos porcentajes se hizo mediante la prueba de ji al cuadrado con la corrección de Yates, o mediante la prueba exacta de Fisher cuando fue necesario.

Los datos de la encuesta sobre conocimientos del vector se digitalizaron en Epi-Info 3.2 y fueron sometidos a validación y limpieza. Esta información se presenta en porcentajes y el análisis de posibles diferencias entre éstos se hizo usando las mismas pruebas aplicadas para los porcentajes de infestación.

\section{Resultados}

\section{Identificación de posibles vectores y algunos aspectos de su biología}

Se identificaron seis especies del género Lutzomyia en los 334 especímenes recolectados en 58 muestras tomadas por todos los métodos de muestreo (cuadro 1). La especie más abundante fue L. longiflocosa Osorno-Mesa, Morales, Osorno \& Hoyos 1970, que correspondió a 80,2\% (268 especímenes) de las capturas. El resto de flebótomos estuvo compuesto por: una especie no identificada del subgénero Helcocyrtomyia $(9,0 \%, n=30)$, L. columbiana (Ristorcelli \& Van Ty 1941$)(7,2 \%, n=24)$, L. nuneztovari (Ortiz 1954) $(1,5 \%, n=5)$, L. trinidadensis (Newstead 1922) $(1,5 \%, n=5)$ y L. shannoni (Dyar 1922) $(0,6 \%$, $\mathrm{n}=2$ ).

Esta amplia dominancia de L. longiflocosa también se mantuvo por sexo, método y sitio de recolección y vereda, incluso en las capturas con cebo humano cuyo número fue limitado (cuadro 1). La mayor abundancia relativa de hembras de $L$. longiflocosa se presentó en la captura con cebo humano en el extradomicilio $(100 \%, n=16)$ y en las capturas con trampas CDC en el intradomicilio $(79,6 \%, n=148)$, en donde $52 \%$ (todas las viviendas de la vereda Tetuancito) había sido fumigado cuatro meses antes del muestreo. Por lo anterior, una buena parte de los resultados del presente estudio se concentran sobre esta especie.

En cuanto al porcentaje de infestación (cuadro 2), se encontró $54,3 \%$ de las viviendas con presencia de flebótomos. De las especies conocidas como antropofílicas, $L$. longiflocosa fue más frecuente $(43,5 \%, 20 / 46)$, seguida de $L$. (Helcocyrtomyia) sp., $(21,7 \%, 10 / 46)$, L. columbiana $(13,0 \%, 6 / 46)$ y $L$. nuneztovari $(6,5 \%, 3 / 46)$. Por municipio, este patrón fue similar. Los porcentajes de infestación para cada una de las especies fueron ligeramente mayores en San Pablo de Ambeima que en Tetuancito. Sin embargo, estas diferencias no fueron estadísticamente significativas.

La abundancia en el intradomicilio de $L$. longiflocosa fue en promedio $0,8 \mathrm{~h} / \mathrm{CDC} / \mathrm{n}$. Por municipio (representado cada uno por la vereda muestreada), el promedio de hembras de $L$. longiflocosa fue, aproximadamente, cinco veces mayor en San Pablo de Ambeima, 1,4 h/CDC/n, comparado con Tetuancito, $0,3 \mathrm{~h} / \mathrm{CDC} / \mathrm{h}$ (cuadro $3)$, diferencia que fue estadísticamente significativa $(z=3,36, p=0,001)$.

Por otro lado, la abundancia de hembras de $L$. longiflocosa varió significativamente de acuerdo con el tipo de hábitat dominante alrededor de las viviendas $\left(\chi^{2}=14,19\right.$, grados de libertad $=5$, $p=0,014$ ) (cuadro 3). El interior de las viviendas rodeadas por "bosque y cafetal tradicional" o por solo cafetal tradicional presentó, aparentemente, las mayores abundancias, $3,9 \mathrm{~h} / \mathrm{CDC} / \mathrm{h}$ y $2,1 \mathrm{~h} /$ $\mathrm{CDC} / \mathrm{n}$, respectivamente. Le siguieron las viviendas rodeadas por "bosque y otros" y los cafetales al sol, ambos con la misma abundancia, $0,8 \mathrm{~h} / \mathrm{CDC} / \mathrm{n}$. La menor abundancia se presentó en las viviendas rodeadas por bosque, $0,4 \mathrm{~h} / \mathrm{CDC} /$ n y "rastrojo o pastizal", 0,1 h/CDC/n. Se confirmó que la abundancia de L. longiflocosa fue significativamente más alta en cafetal tradicional 
Cuadro 1. Composición y abundancia relativa de especies de Lutzomyia capturadas con trampas CDC y cebo humano en las veredas de San Pablo de Ambeima (municipio de Chaparral) y Tetuancito (municipio de San Antonio) en el departamento del Tolima.

\begin{tabular}{|c|c|c|c|c|c|c|c|c|c|c|c|c|c|c|c|c|c|c|c|c|c|}
\hline \multirow{2}{*}{$\begin{array}{l}\text { Método Especie de } \\
\text { (sitio) }^{\mathrm{a}} \text { Lutzomyia }\end{array}$} & \multicolumn{7}{|c|}{ San Pablo de Ambeima } & \multicolumn{7}{|c|}{ Tetuancito } & \multicolumn{7}{|c|}{ Total } \\
\hline & $\mathrm{n}^{\mathrm{b}}$ & q & $\%$ & $\sigma^{*}$ & $\%$ & total & $\%$ & $\mathbf{n}$ & q & $\%$ & $\sigma^{*}$ & $\%$ & total & 1 \% & $\mathbf{n}$ & q & $\%$ & $0^{*}$ & $\%$ & total & $\%$ \\
\hline \multicolumn{22}{|l|}{ CDC } \\
\hline L. longiflocosa* & & 135 & 82,3 & 40 & 88,9 & 175 & 83,7 & & 13 & 59,1 & 4 & 100 & 176 & 65,4 & & 148 & 79,6 & 44 & 89,8 & 3192 & 81,7 \\
\hline Helcocyrtomyia sp." & & 19 & 11,6 & 2 & 4,4 & 21 & 10,0 & & 3 & 13,6 & 0 & & 3 & 11,5 & & 22 & 11,8 & 2 & 4,1 & 24 & 10,2 \\
\hline L. columbiana* & & 2 & 1,2 & 1 & 2,2 & 3 & 1,4 & & 5 & 22,7 & 0 & & 5 & 19,2 & & 7 & 3,8 & 1 & 2,0 & 8 & 3,4 \\
\hline L. nuneztovari" & & 3 & 1,8 & 1 & 2,2 & 4 & 1,9 & & 1 & 4,5 & 0 & & 1 & 3,8 & & 4 & 2,2 & 1 & 2,0 & 5 & 2,1 \\
\hline L. trinidadensis & & 4 & 2,4 & 1 & 2,2 & 5 & 2,4 & & 0 & & 0 & & 0 & & & 4 & 2,2 & 1 & 2,0 & 5 & 2,1 \\
\hline L. shannoni & & 1 & 0,6 & 0 & & 1 & 0,5 & & 0 & & 0 & & 0 & & & 1 & 0,5 & 0 & & 1 & 0,4 \\
\hline Subtotal & & 164 & & 45 & & 209 & & & 22 & & 4 & & 26 & & & 186 & & 49 & & 235 & 70,4 \\
\hline \multicolumn{22}{|l|}{ CDC } \\
\hline L. longiflocosa & & 28 & 65,1 & 25 & 89,3 & 53 & 74,6 & & 3 & 75,0 & 1 & 100 & $4 \varepsilon$ & 80,0 & & 31 & 66,0 & 26 & 89,7 & 57 & 75,0 \\
\hline Helcocyrtomyia sp. & & 1 & 2,3 & 3 & 10,7 & 4 & 5,6 & & 0 & & 0 & & 0 & & & 1 & 2,1 & 3 & 10,3 & 34 & 5,3 \\
\hline L. columbiana & & 14 & 32,6 & 0 & & 14 & 19,7 & & 1 & 25,0 & 0 & & 12 & 20,0 & & 15 & 31,9 & 0 & & 15 & 19,7 \\
\hline Subtotal & & 43 & & 28 & & 71 & & & 4 & & 1 & & 5 & & & 47 & & 29 & & 76 & 22,8 \\
\hline $\begin{array}{l}\mathrm{CH} \\
\text { (intra) }\end{array}$ & 1 & & & & & & & 0 & & & & & & & 1 & & & & & & \\
\hline L. longiflocosa & & 3 & 50,0 & 0 & & 3 & 50,0 & & & & & & & & & 3 & 50,0 & 0 & 3 & & 50,0 \\
\hline Helcocyrtomyia sp. & & 2 & 33,3 & 0 & & 2 & 33,3 & & & & & & & & & 23 & 33,3 & 0 & & 2 & 33,3 \\
\hline L. columbiana & & 1 & 16,7 & 0 & & 1 & 16,7 & & & & & & & & & 1 & 16,7 & 0 & & 1 & 16,7 \\
\hline Sub-total & & 6 & & 0 & & 6 & & & & & & & & & & 6 & & 0 & & 6 & 1,8 \\
\hline $\begin{array}{l}\mathrm{CH} \\
\text { (extra) }\end{array}$ & 0 & & & & & & & 2 & & & & & & & 2 & & & & & & \\
\hline L. longiflocosa & & & & & & & & & 16 & 100 & 0 & & $16 \varsigma$ & 94,1 & & 16 & 100 & 0 & & 16 & 94,1 \\
\hline L. shannoni & & & & & & & & & 0 & & 1 & 100 & 1 & 5,9 & & 0 & & 1 & 100 & 1 & 5,9 \\
\hline Subtotal & & & & & & & & & 16 & & 1 & & 17 & & & 16 & & 1 & & 17 & 5,1 \\
\hline Total & 29 & 213 & & 73 & & 286 & 86 & 29 & 42 & & 6 & & 48 & 14 & 58 & 255 & & 79 & & 334 & \\
\hline
\end{tabular}

${ }^{a} \mathrm{CDC}$ : trampa de luz tipo CDC; CH: cebo humano; intra: intradomicilio; extra: extradomicilio; 'tamaño de muestra; *especies consideradas como antropofílicas

Cuadro 2. Porcentaje de infestación de viviendas con especies de Lutzomyia (capturas con trampas de luz CDC) en las dos veredas muestreadas.

\begin{tabular}{|c|c|c|c|c|c|c|}
\hline \multirow[b]{2}{*}{ Especie de Lutzomyia } & \multicolumn{2}{|c|}{$\begin{array}{l}\text { San Pablo de Ambeima } \\
\qquad\left(n^{a}=22\right)\end{array}$} & \multicolumn{2}{|c|}{$\begin{array}{c}\text { Tetuancito } \\
(n=24)\end{array}$} & \multicolumn{2}{|c|}{$\begin{array}{l}\text { Total } \\
(n=46)\end{array}$} \\
\hline & $n^{b}$ & $\%$ & $\mathbf{n}$ & $\%$ & $\mathbf{n}$ & $\%$ \\
\hline L. longiflocosa* & 10 & 45,5 & 10 & 41,7 & 20 & 43,5 \\
\hline Helcocyrtomyia sp.* & 7 & 31,8 & 3 & 12,5 & 10 & 21,7 \\
\hline L. columbiana* & 3 & 13,6 & 3 & 12,5 & 6 & 13,0 \\
\hline L. nuneztovari* & 2 & 9,1 & 1 & 4,2 & 3 & 6,5 \\
\hline L. trinidadensis & 3 & 13,6 & 0 & & 3 & 6,5 \\
\hline L. shannoni & 1 & 4,5 & 0 & & 1 & 2,2 \\
\hline Total & 12 & 54,5 & 13 & 54,2 & 25 & 54,3 \\
\hline
\end{tabular}

anúmero de viviendas muestreadas; ${ }^{b}$ número de viviendas con presencia de cada especie de Lutzomyia; *especies consideradas como antropofílicas 
Cuadro 3. Abundancia intradomiciliaria (capturas con trampas de luz CDC) de hembras de Lutzomyia longiflocosa según los hábitats dominantes en torno a las viviendas.

\begin{tabular}{|c|c|c|c|c|c|c|c|c|c|c|c|c|}
\hline \multirow{2}{*}{$\begin{array}{l}\text { Hábitat en torno } \\
\text { a la vivienda }\end{array}$} & \multicolumn{4}{|c|}{ San Pablo de Ambeima } & \multicolumn{4}{|c|}{ Tetuancito } & \multicolumn{4}{|c|}{ Total } \\
\hline & $\mathbf{n}^{\mathrm{a}}$ & \& & $\mathbf{M G}^{\mathbf{b}}$ & $\mathrm{IC} 95 \%{ }^{\mathrm{c}}$ & $\mathbf{n}$ & q & MG & IC95\% & $\mathbf{n}$ & ㅇ & MG & IC95\% \\
\hline Bosque & 4 & 2 & 0,3 & $(-0,5-2,2)$ & 3 & 2 & 0,4 & $(-0,7-6,0)$ & 7 & 4 & 0,4 & $(-0,2-1,2)$ \\
\hline Bosque y otros ${ }^{d}$ & 8 & 12 & 0,9 & $(0-2,6)$ & 1 & 0 & 0 & & 9 & 12 & 0,8 & $(0-2,2)$ \\
\hline $\begin{array}{l}\text { Bosque y } \\
\text { cafetal tradicional }\end{array}$ & 2 & 23 & 3,9 & $(-1,0->1000)$ & 0 & & & & 2 & 23 & 3,9 & $(-1->1000)$ \\
\hline Cafetal tradicional & 3 & 78 & 6,2 & $(-1,0->1000)$ & 4 & 4 & 0,7 & $(-0,4-3,8)$ & 7 & 82 & 2,1 & $(-0,3-12)$ \\
\hline Cafetal al sol & 2 & 18 & 3,4 & $(-1,0->1000)$ & 9 & 7 & 0,5 & $(0-1,3)$ & 11 & 25 & 0,8 & $(0-2,4)$ \\
\hline Rastrojo o pastizal & 3 & 2 & 0,4 & $(-0,7-6,0)$ & 6 & 0 & 0 & & 9 & 2 & 0,1 & $(-0,1-0,5)$ \\
\hline Total & 22 & 135 & 1,4 & $(0,4-3,2)$ & $24^{e}$ & 13 & 0,3 & $(0,1-0,6)$ & 46 & 148 & 0,8 & $(0,3-1,4)$ \\
\hline
\end{tabular}

${ }^{a}$ número de viviendas muestreadas; ${ }^{b}$ promedio geométrico del número de hembras/trampa/noche; ${ }^{\circ}$ intervalo de confianza del $95 \% ;$ "otros" se refiere a la presencia de uno o varios de los siguientes hábitats: rastrojo, pastizal, cafetal al sol y caña; e incluye una vivienda sin información sobre tipo de hábitat.

comparada con "bosque y otro" ( $z=-2,66, p=0,008)$, y que la abundancia en "rastrojo o pastizal" era significativamente menor comparada con cafetal tradicional $(z=-2,99, p=0,003)$, con "bosque y cafetal tradicional" $(z=-2,02, p=0,043)$ y con cafetal al sol $(z=2,30, p=0,021)$. De las hembras de $L$. longiflocosa capturadas en el intradomicilio, $30,4 \%(45 / 148)$ se habían alimentado con sangre.

Aunque no se registró la altitud en todas las viviendas, los datos tomados en $35 \%$ (16/46) de éstas permitieron establecer que $L$. longiflocosa se presentaba, por lo menos, entre los 1.300 y los $1.650 \mathrm{msnm}$

No se encontraron evidencias de comportamiento endofílico. El muestreo durante $17 \mathrm{~h}$ (tiempo promedio de muestreo $=22$ minutos/vivienda/2 personas) de los posibles sitios de reposo dio resultados negativos para la presencia de flebótomos.

\section{Conocimientos de la población humana sobre el vector}

El análisis de los conocimientos del insecto vector se hizo con base en 44 encuestas realizadas en 41 viviendas (población=242 habitantes), previa exclusión de cinco encuestas de familias con un tiempo de residencia muy corto ( $<1$ año). Las personas entrevistadas fueron $55 \%$ hombres y $45 \%$ mujeres con una edad mediana de 39 años (cuartil $_{25}=26$, cuartil ${ }_{75}=54$ ) y un tiempo de residencia con una mediana de 9 años $\left(\right.$ cuartil $_{25}=4$, cuartil $_{75}=22$ ).

La gran mayoría de los entrevistados, 95\% (42/ 44), reconocieron, por lo menos, dos de las cinco características que describían a los flebótomos (género Lutzomyia). Los entrevistados reconocieron en promedio 3,8 de las características descritas, con valores muy similares para las dos veredas (cuadro 4).

Todos los habitantes que reconocieron a los flebótomos los conocían por los nombres de "capotillo" o "mantablanca".

La gran mayoria de entrevistados, 73,8\% (31/42), incluyendo aquéllos que mencionaron a la leishmaniasis junto con los síntomas de la picadura, identificaron a los flebótomos como los transmisores de la leishmaniasis. Aunque, por vereda, en San Pablo de Ambeima hubo aparentemente una mayor conciencia de este hecho, 88,9\% (16/18), comparado con Tetuancito, $62,5 \%(15 / 24)$, estas diferencias no fueron significativas.

La vivienda fue señalada por los entrevistados como el sitio en donde se presenta la mayor abundancia, 66,7\% (28/42) de flebótomos, seguida por el cafetal, $45,2 \%$ (19/42), y el bosque, $35,7 \%$ (15/42). Por vereda se observó tendencia a un reconocimiento mayor de los mencionados hábitats en San Pablo de Ambeima (vivienda, 
Cuadro 4. Características de Lutzomyia, presentadas como las de un insecto nocturno que pica, reconocidas por las personas entrevistadas del área de estudio.

\begin{tabular}{|c|c|c|c|c|c|c|c|}
\hline \multirow[b]{3}{*}{ Vereda } & \multicolumn{6}{|c|}{ Características de insecto nocturno que pica (\%) } & \multirow{3}{*}{$\begin{array}{l}\text { Promedio de } \\
\text { características } \\
\text { reconocidas } \\
(I C 95 \%)^{b}\end{array}$} \\
\hline & \multicolumn{3}{|c|}{ Morfológicas } & \multirow{2}{*}{$\begin{array}{c}\text { Desplazamiento } \\
\text { movimiento } \\
\text { en saltos y } \\
\text { vuelo corto }\end{array}$} & \multicolumn{2}{|c|}{ Picadura y su efecto } & \\
\hline & $\mathbf{n}^{\mathbf{a}}$ & $\begin{array}{c}\text { que } \\
\text { zancudo }\end{array}$ & $\begin{array}{c}\text { Alas } \\
\text { paradas }\end{array}$ & & $\begin{array}{c}\text { picadura } \\
\text { como "quemón" }\end{array}$ & $\begin{array}{c}\text { deja "mancha } \\
\text { colorada" }\end{array}$ & \\
\hline \multicolumn{2}{|c|}{$\begin{array}{l}\text { San Pablo } \\
\quad \text { de Ambeima18 }\end{array}$} & 100 & 72,2 & 44,4 & 83,3 & 61,1 & $3,6(2,9-4,3)$ \\
\hline Tetuancito & 24 & 100 & 83,3 & 66,7 & 75,0 & 66,7 & $3,9(3,4-4,4)$ \\
\hline Total & 42 & 100 & 78,6 & 57,1 & 78,6 & 64,3 & $3,8(3,4-4,2)$ \\
\hline
\end{tabular}

a número de entrevistas (una por vivienda); ${ }^{\text {b }}$ intervalo de confianza del $95 \%$

$72,2 \%$ (13/18); cafetal y bosque, $55,5 \%$ (10/18), cada uno) que en Tetuancito (vivienda, 62,5\% (15/ 24); cafetal, $37,5 \%$ (9/24), y bosque, $20,8 \%$ (5/ 24)). Sin embargo, esta diferencia sólo se confirmó estadísticamente para el caso del bosque $\left(\chi^{2}=3,99, p=0,046\right)$.

En cuanto a las épocas de mayor abundancia del género Lutzomyia, la mayoría de entrevistados, $83,3 \%$ (35/42), identificó ambas o una de las dos épocas secas del año como las de mayor abundancia. Por vereda, el porcentaje de entrevistados que reconoció estas épocas fue ligeramente mayor en San Pablo de Ambeima, $88,9 \%$ (16/18) comparado con Tetuancito, $79,2 \%$ (19/24), pero sin diferencias significativas.

\section{Opinión sobre el posible uso de toldillos para el control de leishmaniasis cutánea}

En cuanto al posible uso de toldillos por parte de la población para el control de la leishmaniasis cutánea, se encontró -con base en las encuestas aplicadas en San Pablo de Ambeima- que 36,8\% (7/19) de los entrevistados había usado toldillos alguna vez; 94,7\% (18/19) manifestó que usarían toldillos si les protegía de la leishmaniasis y $83,3 \%(15 / 18)$ de éstos no vio ningún inconveniente en que los toldillos estuvieran impregnados con insecticida.

\section{Discusión}

En el presente estudio se encontraron cuatro especies antropofílicas que podrían ser importantes desde el punto de vista epidemiológico:
L. Iongiflocosa, L. columbiana, L. nuneztovari y una especie no identificada del subgénero Helcocyrtomyia. Las tres primeras especies pertenecen al grupo verrucarum, reconocido por incluir varias especies endémicas de la zona montañosa de la Región Andina, adaptadas a vivir en cultivos de café e involucradas como vectores probables o confirmados de leishmaniasis cutánea o bartonelosis (5).

De las especies antropofílicas identificadas, los resultados indican que $L$. longiflocosa, especie endémica en Colombia, distribuida principalmente en la región subandina de la cuenca alta del río Magdalena y en un área limitada de la cuenca media (9), podría ser el vector principal de la leishmaniasis cutánea en el área de estudio. Esto se sustenta en que $L$. longiflocosa fue ampliamente dominante en las capturas por todos los métodos de muestreo y presentó la mayor endofagia (presencia en $43,5 \%$ de las viviendas muestreadas con trampas CDC).

La endofagia de L. longiflocosa se confirmó indirectamente en la encuesta sobre conocimientos del vector, en la cual la mayoría de los entrevistados, $66,7 \%$, señaló la vivienda como uno de los sitios de mayor abundancia de los flebótomos. Este comportamiento endofágico de $L$. longiflocosa apoya la presunción de que una parte importante de la transmisión se está dando en el intradomicilio (2).

Por otro lado, con los mismos criterios de dominancia y endofagia, $L$. longiflocosa ha sido 
involucrada como posible vector (probablemente de L. braziliensis) en tres focos de leishmaniasis cutánea en Colombia, localizados también sobre la región subandina. El primer foco incluye los municipios de Baraya, Tello, Neiva, en el departamento del Huila, en donde ocurrió una epidemia entre 1993 y 1996 con 1.249 casos. El segundo foco ubicado en el municipio de Planadas (Tolima) presentó un pequeño brote en 1998 en el que se registraron 86 casos (3). El tercer foco, localizado en el municipio de Ábrego (Norte de Santander) presentó también un pequeño brote con 102 casos entre los años 2002 y 2004 (9).

Además, en el foco del Huila se demostró que la mayor abundancia de L. longiflocosa coincidió con el área geográfica en donde se localiza el foco (Pardo R, Ferro C, Lozano G, Lozano C, Cabrera OL, Davies C. Flebótomos (Diptera:Psychodidae) vectores de leishmaniasis cutánea y sus determinantes ecológicos en la zona cafetera del departamento del Huila. Memorias, XXVI Congreso Sociedad Colombiana de Entomología, Bogota 2830 de julio de 1999) y se encontró una asociación estadística entre la abundancia intradomiciliaria de L. longiflocosa y la prevalencia acumulada de leishmaniasis cutánea en las viviendas (información de los autores, sin publicar).

Finalmente, en un estudio de laboratorio se logró la infección experimental de L. longiflocosa con L. braziliensis y la transmisión del parásito por picadura a un hospedero experimental (10). Estas evidencias apoyan la propuesta de que $L$. longiflocosa es el más probable vector de leishmaniasis cutánea en el foco de Chaparral y San Antonio.

Con relación a las otras tres especies, $L$. columbiana, L. nuneztovari y L. (Helcocyrtomyia) sp., su relativa baja abundancia en las capturas con trampas CDC en el intradomicilio (entre 3,4\% y $10,2 \%$ ) y el extradomicilio (entre 0 y $19,7 \%$ ), y la baja infestación de las viviendas (entre 6,5\% y $21,7 \%$ ) limitan sus posibilidades como vectores. Por esto se considera que podrían jugar solamente un papel secundario en la transmisión de la leishmaniasis cutánea. De estas especies de flebótomos, las dos primeras presentan antecedentes epidemiológicos.
L. columbiana, otra especie endémica de Colombia, tiene una distribución más amplia que la de L. longiflocosa; se distribuye en varias localidades a lo largo de la región subandina de las tres cordilleras $(5,6)$. Esta especie se considera el probable vector de Leishmania mexicana en el municipio de Samaniego, Nariño $(11,12)$ y de $L$. braziliensis y $L$. panamensis en el municipio de Dagua, Valle del Cauca (13), focos en donde se reportaron menos de 30 casos de leishmaniasis cutánea en cada uno.

L. nuneztovari es una especie de amplia distribución, presente en la mayoría de la Región Andina (Bolivia, Perú, Colombia y Venezuela) y en Centroamérica (Guatemala, Honduras y Panamá) (5). Aunque en Colombia esta especie no ha sido involucrada como vector principal en ningún foco de leishmaniasis cutánea, algunos especímenes de esta especie se infectaron experimentalmente con L. braziliensis (14). En Bolivia, la subespecie $L$. nuneztovari anglesi es considerada vector de $L$. braziliensis en el foco de Las Yungas $(15,16)$ y de $L$. amazonensis en Cajuata (17).

Finalmente, con relación a $L$. (Helcocyrtomyia) $\mathrm{sp}$., las dificultades para identificar las especies del subgénero Helcocyrtpmyia también se han presentado en los focos de Baraya, Tello y Neiva (Huila) y en el de Planadas (Tolima). Algunas especies de este subgénero han sido involucradas como vectores de leishmaniasis cutánea en la región subandina. $L$. hartmanni es vector de $L$. columbiensis en Colombia (18). L. peruensis y $L$. ayacuchensis son vectores de $L$. peruviana en Perú (19).

A pesar de la relativa cercanía, $55 \mathrm{~km}$ entre las dos veredas estudiadas, la abundancia intradomiciliaria de $L$. longiflocosa fue significativamente mayor en San Pablo de Ambeima, municipio de Chaparral, 1,4 h/CDC/n, comparada con Tetuancito, municipio de San Antonio, $0,3 \mathrm{~h} / \mathrm{CDC} / \mathrm{n}$. Este resultado puede tener varias explicaciones. Inicialmente, la fumigación en Tetuancito (cuatro meses antes del muestreo) pudo ser la responsable de la diferencia. Sin embargo, las capturas con trampa CDC en el extradomicilio, en donde es difícil que la 
fumigación intradomiciliaria tenga impacto, sugieren también una mayor abundancia de $L$. longiflocosa $(n=53)$ en San Pablo de Ambeima que en Tetuancito $(n=4)$. Además, la falta de comportamiento endofílico permiten suponer que la fumigación tendría un impacto muy limitado sobre $L$. longiflocosa, como se explicará más adelante.

Otra posible explicación sería algunas diferencias climáticas entre los dos municipios. En particular, la precipitación anual en Chaparral es mayor, $2.607 \mathrm{~mm}$, que en San Antonio, $1.985 \mathrm{~mm}$. Si se acepta que la fumigación en Tetuancito no afectó la abundancia intradomiciliaria de L. Iongiflocosa, entonces, la mayor abundancia de esta especie en Chaparral podría explicar, al menos en parte, la mayor incidencia de leishmaniasis cutánea en este municipio comparada con San Antonio.

Con relación a la variación en abundancia intradomiciliaria, según el tipo de hábitat circundante a las viviendas, los resultados entomológicos confirman que los hábitats con mayor riesgo de contacto con L. longiflocosa (y otras especies vectoras) son los bosques y los hábitats de estructura similar como el cafetal de tipo tradicional en donde $L$. longiflocosa presenta sus mayores abundancias (Pardo R, Ferro C, Lozano G, Lozano C, Cabrera OL, Davies C. Flebótomos (Diptera: Psychodidae) vectores de leishmaniasis cutánea y sus determinantes ecológicos en la zona cafetera del departamento del Huila. Memorias, XXVI Congreso Sociedad Colombiana de Entomología, Bogotá, 28 al 30 de julio de 1999) (20). Esto coincide con las observaciones de los habitantes del área de estudio quienes reconocieron el cafetal y el bosque, junto con las viviendas, como los sitios en donde los flebótomos presentan alta abundancia.

A pesar de que no se cuenta con evidencias entomológicas que lo confirmen, hubo una amplia concordancia entre los habitantes, $83,3 \%$ de los entrevistados, en que los flebótomos presentan una mayor abundancia en las épocas de verano o de reducción de lluvias. Esta misma observación fue hecha por los habitantes en el foco de $L$. longiflocosa en el departamento del Huila (Pardo $\mathrm{RH}$, Carvajal A, Ferro C, Davies C. Effect of knowledge and economic status on sandfly control activities by householders at risk of cutaneous leishmaniasis in the subandean region of Huila department, Colombia, Biomédica, en prensa). Lo anterior sugiere que $L$. longiflocosa podría ser una "especie de época seca", lo que parece ser común con otros vectores sospechosos en el grupo verrucarum en la zona montañosa andina, por ejemplo, L. columbiana en Samaniego, Nariño (11) y L. youngien La Guaira, Valle del Cauca (14). Lo anterior indica que las épocas de verano son las de mayor riesgo de contacto con el vector $y$, posiblemente, de mayor riesgo de transmisión. Sin embargo, lo último debe tomarse con cautela pues se ha observado que una mayor transmisión puede ocurrir también cuando la densidad del vector disminuye y aumenta la población de hembras paridas (21).

Con relación a la distribución altitudinal de $L$. longiflcosa, los registros del presente estudio 1.300 a 1.650 msnm- aunque limitados, están dentro del rango de distribución detectado (940$2.090 \mathrm{msnm}$ ) para esta especie (Pardo R, Ferro C, Lozano G, Lozano C, Cabrera OL, Davies C. Flebótomos (Diptera: Psychodidae) vectores de leishmaniasis cutánea y sus determinantes ecológicos en la zona cafetera del departamento del Huila. Memorias, XXVI Congreso Sociedad Colombiana de Entomología, Bogotá, 28 al 30 de julio de 1999), queda pendiente confirmar si la altitud de mayor riesgo es, también, similar a la detectada, $1.300-1.700 \mathrm{msnm}$, en el foco del departamento del Huila (Pardo R, Ferro C, Lozano G, Lozano C, Cabrera OL, Davies C. Flebótomos (Diptera: Psychodidae) vectores de leishmaniasis cutánea y sus determinantes ecológicos en la zona cafetera del departamento del Huila. Memorias, XXVI Congreso Sociedad Colombiana de Entomología, Bogotá, 28 al 30 de julio de 1999)

El conocimiento sobre los flebótomos y su papel en la transmisión de la leishmaniasis cutánea es fundamental para la aplicación de medidas de control. Si los flebótomos no son considerados como importantes en la transmisión por la población afectada, ésta puede no tomar las medidas de protección adecuadas (22). Lo anterior es particularmente importante para el caso de las 
medidas en las que se requiere una participación activa de la comunidad, como es el caso de los toldillos impregnados con insecticida.

Los resultados del presente estudio, aunque representan una aproximación al conocimiento que los habitantes tienen sobre el vector y la leishmaniasis cutánea, deben tomarse con precaución pues el conocimiento puede estar sobreestimado. Esto como consecuencia de que en las viviendas seleccionadas para el estudio sólo se incluyeron familias afectadas por la enfermedad que, se presume, estuvieron más expuestas al contacto con el vector, así como a las campañas de información realizadas por las entidades de salud.

La población reconoce muy bien las Lutzomyia, 95\% de los entrevistados, llamándolos "capotillo" - "mantablanca", nombre común en los departamentos de la cuenca alta del río Magdalena (23). Esto indica que la abundancia de flebótomos $y$, probablemente, el contacto humano-vector son altos. Se ha demostrado que las personas residentes en los sitios con alta abundancia de flebótomos tienen un mayor conocimiento de estos insectos (20).

El alto conocimiento, $73,8 \%$, del papel de los flebótomos como vectores de la leishmaniasis cutánea se puede atribuir a un éxito en las campañas educativas realizadas por las secretarías de salud departamental y municipales y al estrecho contacto humano-vector, gran parte del cual se da en el intradomicilio. Sin embargo, este conocimiento puede, también, deberse en buena parte a la sensibilización de la comunidad por lo reciente de la epidemia y por su magnitud. Por el contrario, en áreas en las que la enfermedad tiene carácter endémico y la mayoría de la transmisión ocurre en el extradomicilio como, por ejemplo, en Chocó, el conocimiento del papel de los vectores ha sido muy bajo (24).

Todo lo anterior indica que $L$. longiflocosa es el probable vector de leishmaniasis cutánea de mayor importancia en el área subandina (entre 1.000 y $2.000 \mathrm{msnm}$, aproximadamente) de la cuenca del río Magdalena, y que presenta un patrón de comportamiento similar a lo largo de ella: a) especie antropofílica dominante; b) comporta- miento endofágico; c) no presenta comportamiento endofílico evidente; c) presenta mayores abundancias en bosque y hábitats con estructura similar; d) aparentemente es una especie de estación seca, y e) la transmisión parece darse tanto en el extradomicilio como en el intradomicilio.

Es de resaltar que esta situación se limita, probablemente, sólo al área montañosa media pues en el piedemonte de la cuenca por debajo de $1.000 \mathrm{msnm}$, el ciclo epidemiológico de la leishmaniasis cutánea, por lo menos en lo que corresponde al departamento del Tolima, es diferente. En esta zona, la especie vectora confirmada es $L$. trapidoi que se ha encontrado infectada naturalmente con $L$. panamensis $(25,26)$. En este ambiente la transmisión intradomiciliaria parece ser menos importante que en el área de estudio.

Los resultados del presente estudio permiten hacer varias observaciones con relación a las medidas, principalmente, la fumigación intradomiciliaria que se ha aplicando para controlar la epidemia. Los altos porcentaje de infestación de las viviendas y el no haber encontrado evidencias de comportamiento endofílico indican que, aunque el control intradomiciliario puede ser efectivo, la utilidad de la fumigación podría verse muy limitada ya que esta medida es particularmente efectiva en especies de comportamiento endofílico, como se ha demostrado para $L$. verrucarum en Perú (8).

En especies de comportamiento exofílico (aquéllas que entran a la vivienda, se alimentan y la abandonan antes $\mathrm{o}$ al amanecer) la fumigación ha fallado en reducir su abundancia intradomiciliaria, como se demostró con $L$. nuneztovari en Bolivia (27). Teniendo en cuenta que $L$. longiflocosa tiene aparentemente comportamiento exofílico en el área de estudio, es muy probable que el uso de toldillos impregnados con insecticida sea una medida más adecuada que la fumigación. Por otro lado, esta medida no tendría problemas de aceptabilidad por parte de la comunidad debido a la buena predisposición al uso de toldillos que se pudo percibir por parte de los habitantes.

La utilidad potencial de los toldillos impregnados para el control de L. longiflocosa se probó en el 
foco de leishmaniasis cutánea en el departamento del Huila en donde los estudios de eficacia y efectividad de los toldillos impregnados mostraron que éstos reducen: a) la actividad de picadura intradomiciliaria, b) el porcentaje de hembras con sangre, la cantidad de sangre tomada y la proporción de hembras con sangre humana (Pardo $R$, Ferro C, Davies C. Efficacy and efectiveness of insecticide treated bednets in Colombia. 5th International Symposium on Phlebotominae sandflies (ISOPS 5) Tunisia, April 17-21, 2005). En este mismo estudio, al evaluar la efectividad de la fumigación intradomiciliaria, los resultados no fueron concluyentes.

La acentuada variación estacional en la abundancia de $L$. longiflocosa, reportada por los entrevistados, puede usarse de manera provisional como guía en la toma de decisiones en cuanto a la temporalidad de la aplicación de medidas de control vectorial y optimizar así el uso de los recursos disponibles.

\section{Agradecimientos}

Los autores agradecen a la Secretaría de Salud Departamental del Tolima, a las alcaldías de los municipios de Chaparral y San Antonio y a los hospitales San Juan Bautista de Chaparral y San Roque de San Antonio por el apoyo logístico; a Diego Morales y a Eduardo Alfonso Lozano Guarín por facilitar el acceso a la información epidemiológica básica y participar en la coordinación de parte del trabajo de campo; a Álvaro Galindo, por su participación en la aplicación de encuestas.

\section{Conflicto de interés}

Los autores declaran que no existe ningún tipo de interés que pudiere influir en los resultados del estudio.

\section{Financiación}

El estudio fue financiado por el Instituto Nacional de Salud y Yale University, proyecto "Filogeografía y genética del grupo Verrucarum". Raúl Pardo es estudiante de Ph.D. con apoyo financiero del Instituto Colombiano para el Desarrollo de la Ciencia y la Tecnología Francisco José de Caldas, Colciencias.

\section{Referencias}

1. Padilla JC, Guhl NF, Soto MJ, Álvarez UG. Diagnóstico y terapéutica de de las enfermedades transmitidas por vectores en Colombia. Santafé de Bogotá, D.C.: Sociedad Colombiana de Parasitología y Medicina Tropical; 1999. p.128.

2. Morales DF, Castaño CS, Lozano EA, Vallejo HJ. Descripción de la epidemia de leishmaniasis cutánea en Chaparral y San Antonio, 2003 y 2004 (semana 24). Inf Quinc Epidemiol Nac 2004;9:177-92.

3. Cárdenas R, Romo GM, Santamaria E, Bello F, Ferro C. Lutzomyia longiflocosa (Diptera: Psychodidae) posible vector en el foco de leishmaniasis cutánea del municipio de Planadas, zona cafetera del Tolima. Biomédica 1999;19: 239-44.

4. Sierra D, Vélez ID, Uribe S. Identificación de Lutzomyia spp. (Diptera: Psychodidae) grupo verrucarum por medio de microscopía electrónica de sus huevos. Rev Biol Trop 2000;48:615-22.

5. Young DG, Duncan MA. Guide to the identification and geographic distribution of Lutzomyia sand flies in México, West Indies, Central and South America (Diptera: Psychodidae). Mem Amer Entomol Inst 1994;54:1-881

6. Bejarano EE, Sierra D, Veléz ID. Novedades en la distribución geográfica del grupo verrucarum (Diptera: Psychodidae) en Colombia. Biomédica 2003;23:34150.

7. Clements AN. The biology of mosquitoes. Wallingford: CABI Publishing; 1999. p.458-79.

8. Davies CR, Llanos-Cuentas EA, Campos P, Monje J, Leon E, Canales J. Spraying houses in the Peruvian Andes with lambda-cyhalothrin protects residents against cutaneous lesihmaniasis. Trans R Soc Trop Med Hyg 2000;94:631-6.

9. Cárdenas R, Pabón E, Anaya H, Sandoval C. Presencia de Lutzomyia longiflocosa (Diptera: Psychodidae) en el foco de leishmaniasis tegumentaria americana del municipio de Ábrego, Norte de Santander. Primer registro para el departamento. Clon, Revista Institucional de la Facultad de Salud de la Universidad de Pamplona 2005;3:7-14.

10. Santamaría E, Castillo M, Cárdenas E, Bello F, Ayala M, Ferro C. Transmisión experimental de Leishmania braziliensis a hámster por picadura de Lutzomyia longiflocosa (Diptera: Psychodidae) provenientes de un foco endémico en la zona cafetera colombiana. Médicas UIS 1998;12:279-84.

11. Montoya-Lerma J, Cadena H, Segura I, Travi BL. Association of Lutzomyia columbiana (Diptera: Psychodidae) with a leishmaniasis focus in Colombia due to species of the Leishmania mexicana complex. Mem Inst Oswaldo Cruz 1999;94:277-83. 
12. Arroyo CG, Garzón J. Investigación de un foco de leishmaniasis cutánea en la zona Andina del departamento de Nariño. Biomédica 1996;16:25-31.

13. Montoya J, Jaramillo C, Palma G, Gomez T, Segura I, Travi B. Report of an epidemic outbreak of tegumentary leishmaniasis in a coffee-growing area of Colombia. Mem Inst Oswaldo Cruz 1990;85:119-21.

14. Santamaría E, Castillo M, Cardenas R, Bello F, Ayala $\mathbf{M}$, Ferro $\mathbf{C}$. Competencia vectorial de las especies de Lutzomyia del grupo verrucarum (Diptera: Psychodidae) en un foco endémico de Leishmania braziliensis en Reventones, Cundinamarca. Biomédica 1999;19:11526.

15. Torres M, Lopez M, Le Pont F, Martinez E, Munoz M, Hervas $D_{\text {; }}$ et al. Lutzomyia nuneztovari anglesi (Diptera: Psychodidae) as a probable vector of Leishmania braziliensis in the Yungas, Bolivia. Acta Trop 1998;71:311-6.

16. Torres JM, Le Pont F, Mouchet J, Desjeux P, Richard A. Epidemiologie de la leishmaniose tegumentaire en Bolivie. 1. Description des zones d'etude et frequence de la maladie. Ann Soc Belge Med Trop 1989;69:297-306.

17. Martinez E, Le Pont F, Torres M, Telleria J, Vargas F, Dujardin JC et al. Lutzomyia nuñeztovari anglesi (Le Pont \& Desjeux, 1984) as a vector of Leishmania amazonensis in a sub-Andean leishmaniasis focus of Bolivia. Am J Trop Med Hyg 1999;61:846-9.

18. Kreutzer RD, Corredor A, Grimaldi G Jr, Grogl M, Rowton ED, Young DG et al. Characterization of Leishmania colombiensis sp.n. (Kinetoplastida: Tripanosomatidae), a new parasite infecting humans, animals, and phlebotomine sand flies in Colombia and Panama. Am J Trop Med Hyg 1991;44:662-75.

19. Dujardin JC, Llanos-Cuentas A, Caceres A, Arana M, Dujardin JP, Guerrini F et al. Molecular karyotype variation in Leishmania (Viannia) peruviana: Indication of geographical populations in Peru distributed along a north-south-cline. Ann Trop Med Parasitol 1993; $87: 335-47$.
20. Alexander B, Agudelo LA, Navarro F, Ruiz F, Molina J, Aguilera $\mathbf{G}$ et al. Phlebotomine sandflies and leishmaniasis risks in Colombian coffee plantations under two systems of cultivation. Med Vet Entomol 2001;15:364-73.

21. Scorza JV, Marquez M, Marquez JC. Hallazgo de Lutzomyia townsendi (Ortiz, 1959) naturalmente infectada con Leishmania braziliensis, en el área suburbana de Trujillo, Venezuela. Bol Dir Malariol San Amb1984;24:21-8.

22. Koirala S, Parija SC, Karki P, Das ML. Knowledge, attitudes, and practices about kala-azar and its sandfly vector in rural communities of Nepal. Bull World Health Org 1998;76:485-90.

23. Montoya J, Ferro C. Flebótomos (Diptera: Psychodidae) de Colombia. En: Amat G, Andrade MG Fernández F, editores. Insectos de Colombia II. Bogotá: Academia Colombiana de Ciencias Exactas, Físicas y Naturales. Colección Jorge Álvarez Lleras No. 13; 1999. p.211-45.

24. Isaza DM, Restrepo BN, Arboleda M, Casas E, Hinestroza H, Yurgaqui T. La leishmaniasis conocimientos y prácticas en poblaciones de la Costa del Pacifico de Colombia. Rev Panam Salud Pub 1999;6:177-84.

25. Morales A, Corredor A, CÁceres E, lbagos AL, Rodríguez C. Aislamiento de tres cepas de Leishmania a partir de Lu. trapidoi en Colombia. Biomédica 1981;1:198-207.

26. Young DG, Morales A, Kreutzer RD, Alexander JB, Corredor A, Tesh RB et al. Isolation of Leishmania braziliensis (Kinetoplastida: tripanosomatidae) from cryopreserved Colombian sand flies (Diptera: Psychodidae). J Med Entomol 1987;24:587-9.

27. Le Pont F, Padilla JM, Desjeux P, Richard A, Mouchet J. Impact de pulverisations de deltamethrine dans un foyer de leishmaniose de Bolivie. Ann Soc Belge Med Trop 1989;69:223-32. 